\title{
OPERATION AND MAINTENANCE OF BUILT ASSETS: A PATHWAY TOWARDS INDIVIDUALISED TRAINING EXPERIENCES
}

\author{
James Wakefield $^{1}$, and Mohamad Kassem ${ }^{2}$
}

\section{Background and Identification of Problem / Knowledge GaP}

Workforce health and safety (H\&S) in the construction sector is a global concern. The construction sector has one of the highest fatal injury rates in both the developed and the developing world (Kassem et al., 2017). Efforts to improve the H\&S performance in the construction sector are plentiful. However, until recently studies have mainly focused on $\mathrm{H} \& \mathrm{~S}$ issues on the construction site. Facilities management and in particular the operation and maintenance of built assets has a much higher rate of injury and illness when compared to all other fields of employment (Wetzel and Thabet, 2018). More effort should be dedicated to address H\&S risks at this stage. Safety learning and training, as a proactive strategy to improve the $\mathrm{H} \& \mathrm{~S}$ performance of the construction sector, has received significant attention in recent years.

Studies proposing new approaches for H\&S learning and training have proposed a number of approaches that are characterized by a varying degree of 'virtualisation' ranging from pure reality, through augmented and mixed reality, to full virtual reality. While the degree of 'virtualisation' of the proposed approaches is an important variable to consider, the extent of 'personalisation' enabled by such approaches is key. Personalisation in learning and training refer to experiences which are tailored to the specific needs of the individual according the job role and to the specific characteristics of the context (company or otherwise) in which the individual is required to complete his/her tasks. This emerging trend is being driven by innovators on both the demand side (e.g. forward-looking clients and operators innovating in health H\&S learning and training to meet ambitious H\&S performance) and on the supply side (e.g. specialised learning and training providers, and technology providers). This paper presents the outcome from the first stage of a study aiming to develop an individualised training solution for the construction and facilities management (FM) domain.

\section{Research Aim And Methodology}

This research aims to establish an individualised solution for $\mathrm{H} \& \mathrm{~S}$ compliance training management and delivery for the construction and facilities management domains. This includes the development of the framework, the corresponding software prototype, and its testing.

The research starts off by adopting a technology-agnostic approach and concentrates on addressing preliminary concepts such as: understanding the importance of individualised training; what makes training individualised; and what are the interplays between personalisation of training and enabling technologies. Following this initial stage, the next step is to develop the conceptual framework for individualised training, the technical specific, the prototype platform, and its testing.

To date, three key knowledge gaps were identified: i) the lack of a framework road-mapping the levels of learning and training experiences and recognising the need for individualised experiences; ii) a consensus as to what makes a learning and training experience individualised and the role of technology in enabling the varying degrees of personalisation; ; and iii) limited availability of digitally-enabled solutions providing individualised learning and training experiences and evidence of their impact on safety performance and productivity.

This paper addresses the first gap by focussing on the H\&S compliance training management and delivery as a specific area of application. Case building (also referred to as 'case writing') was used to solicit knowledge about current practice and methods of compliance training management and delivery through interviews of five experts from the construction and facilities management (FM) who are involved in compliance training delivery and management. The knowledge from the interviews was assimilated into a pathway showing the trajectory of $\mathrm{H} \& \mathrm{~S}$ training perspectives including the different levels of personalisation and their interplay with data and interoperability of the enabling technologies. To help the knowledge assimilation exercise, use cases covering different FM perspectives including multiple scenarios (e.g. Trainee Environmental Surveyor responsible for undertaking surveys in the built environment

\footnotetext{
${ }^{1} \mathrm{PhD}$ Candidate, Department of Mechanical and Construction Engineering, Northumbria University, Newcastle, UK

${ }^{2}$ Associate Professor, Department of Mechanical and Construction Engineering, Northumbria University, Newcastle, UK
} 
undertaking surveys in the built environment to test for the presence of asbestos with/without credentials to access site, etc.) were explored. The pathway is presented in the next section.

\section{RESEARCH FINDINGS}

The pathway towards individualised training is included in Figure 1. It shows the trajectory from a generalised training towards a fully individualised training offer. The generalised training offer represents arrangements which are common in the UK for facilities management site operatives. For example, prior to accessing the site (and as a condition of the Service Level Agreement between client and contractor) the operative is required to have undertaken regulatory and industry standard training in order to be deemed suitably qualified (and as such insured) to undertake the task in hand.

Beyond the generalised domain, training has the potential to become individualised at three main levels: i) Baseline individualised represents training related to general client arrangements such as client process and procedures around a specific risk; ii) Semi-individualised: addresses training which is specific to the site on which the job is being carried out; and iii) fully individualised: covers site (e.g. specific location/condition of the site), type of job/task (e.g. asbestos removal) and individual (e.g. credential of the specific individuals undertaking the job) specific training.

The fundamental challenge when moving to higher levels of individualisation is in the interoperability of systems which currently are commonly existing in silo (e.g. systems for scheduling, HR, job sheets, training plans).

The pathway proposes that the more interoperable are the systems holding data regarding job scheduling, built asset data (including risk classification) and training plans (including outcomes), the more likely are the possibilities for realising a progressive individualised training offer. Other important aspects that change when moving towards individualised training are 'data' and 'interoperability'. Data type and data requirements change and increase for higher level of individualisations. Data type change from static to dynamic type which bring challenges to its change management to ensure that the right and most up-to-date information is used in the individualised training. Also the volume of the required data from across disparate systems increases when moving towards higher level of personalisation. This pathway is put forward in the context of the H\&S compliance training delivery and management but it can be applied to other areas of training. The next step in this research is to establish and validate a framework and a technical specification for fully individualised training approach, and develop and test the platform prototype.

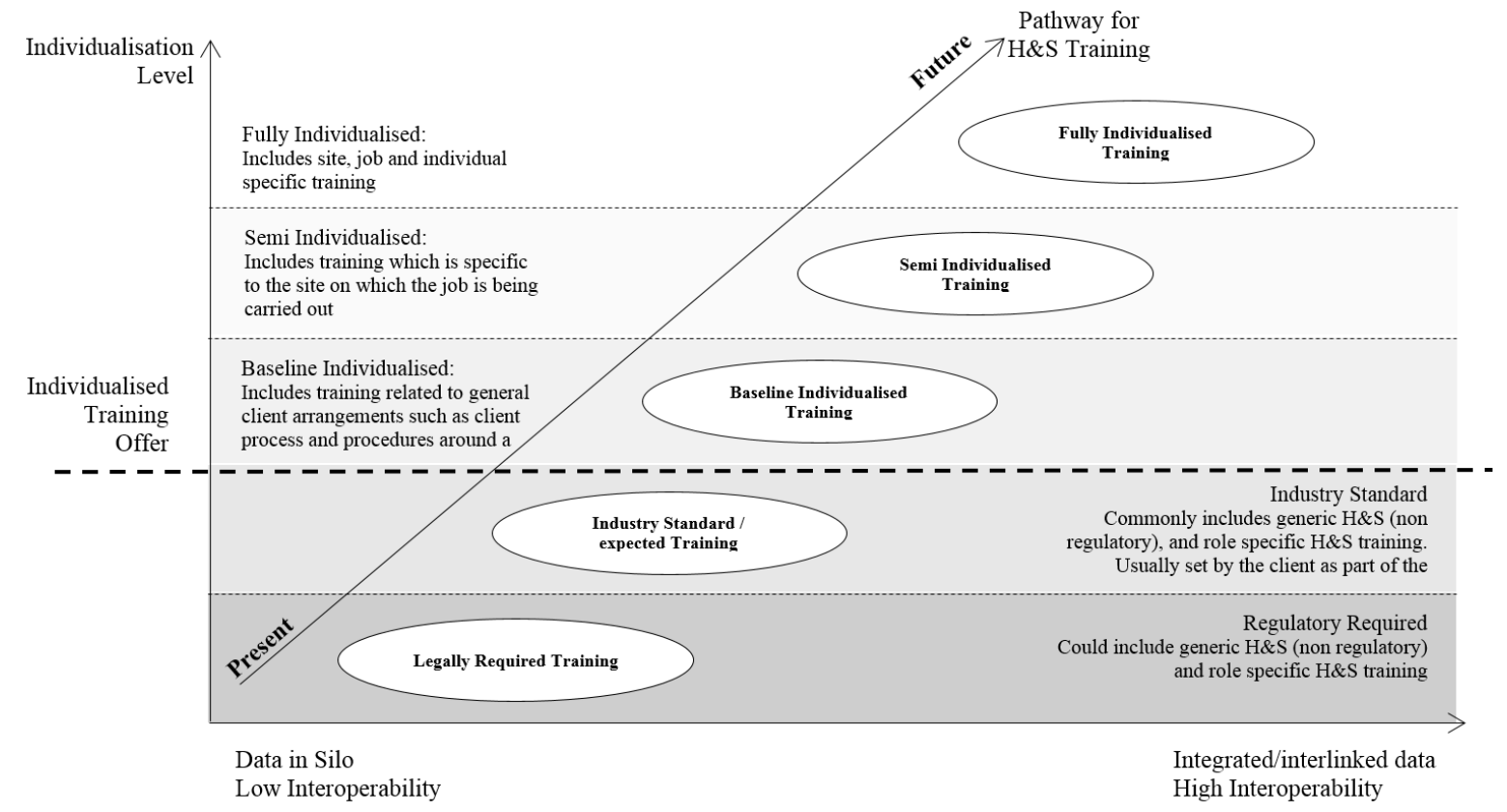

Figure 1: Towards Individualised Training: A Health and Safety Training Perspective in the FM Domain

\section{REFERENCES}

Wetzel, E.M. and Thabet, W. Y. (2018). A case study towards transferring relevant safety information for facilities maintenance using BIM. Journal of Information Technology in Construction (ITcon), Vol. 23, pg. 53-74, http://www.itcon.org/2018/3

Kassem M., Benomran L., Teizer J. Virtual environments for safety learning in construction and engineering: seeking evidence and identifying gaps for future research, Visualization in Engineering, Springer, 5:16, 2017, http://doi.org/10.1186/s40327-017-0054-1 